\title{
Clinical phenotype and outcomes of pneumococcal versus meningococcal purpura fulminans: a multicenter retrospective cohort study
}

\author{
Damien Contou ${ }^{1^{*}}$ (D) and Nicolas de Prost $^{2}$ on behalf of the HOPEFUL Study group
}

Keywords: Purpura fulminans, Neisseria meningitidis, Streptococcus pneumoniae, Septic shock, Meningitis

Purpura fulminans (PF) is a rare cause of septic shock characterized by the association of a sudden and extensive purpuric rash together with an acute circulatory failure [1] leading to high rates of intensive care unit (ICU) mortality [1,2] and long-term sequelae [3]. Clinical presentation of patients with PF differs from that of patients with meningitis since PF patients are commonly admitted to the ICU for hemodynamic impairment exposing them to early death from refractory circulatory failure, as opposed to patients with meningitis who are usually admitted to the ICU for neurological impairment. Among adult patients, Neisseria meningitidis and Streptococcus pneumoniae are the most commonly involved microorganisms accounting for more than $80 \%$ of PF [1] and meningitis [4]. While clinical features and outcomes widely differ between adult patients with pneumococcal and meningococcal meningitis [4], it remains unclear whether pneumococcal (pPF) and meningococcal (mPF) PF exhibit different clinical phenotypes and outcomes, although pPF was previously shown to predominantly occur in asplenic patients [5] and carries a higher risk of limb amputation [1]. We therefore compared the clinical, biological presentations and outcome of adult patients with $\mathrm{pPF}$ and $\mathrm{mPF}$.

*Correspondence: damien.contou@ch-argenteuil.fr

1 Service de Réanimation Polyvalente, Centre Hospitalier Victor Dupouy, 69, rue du Lieutenant-Colonel Prudhon, 95100 Argenteuil, France

Full list of author information is available at the end of the article
We performed an ancillary analysis of a 17-year multicenter retrospective study conducted in 55 centers in France, which included all consecutive patients ( $\geq 18$ years) admitted to the ICU for an infectious PF (2000-2016) [1]. Patients with non-microbiologically documented PF or a bacterial documentation other than Neisseria meningitidis and Streptococcus pneumoniae were excluded.

During the study period, 195 patients with mPF and 67 with pPF were included. As compared to patients with $\mathrm{mPF}$, those with pPF were older and had higher ICU severity scores. Chronic alcoholism and asplenia were more frequent in $\mathrm{PPF}$, while the proportion of patients without previous comorbid conditions was lower. The time elapsed between disease onset and ICU admission was longer and purpura was less often noticed before ICU admission in pPF than in mPF. pPF patients also had lower platelet counts, higher serum urea and creatinine levels, and more frequent bacteremia. pPF patients needed more frequent invasive mechanical ventilation support, renal replacement therapy, plasma and platelets transfusions and had higher durations of invasive mechanical ventilation and vasopressor support. ICU mortality and rate of limb amputation were higher in patients with pPF (Table 1).

The Kaplan-Meier survival analysis did not show significant difference between $\mathrm{pPF}$ and $\mathrm{mPF}$ patients $(p=0.80$ by the log-rank test, Fig. 1$)$.

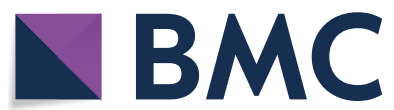

(c) The Author(s) 2021. Open Access This article is licensed under a Creative Commons Attribution 4.0 International License, which permits use, sharing, adaptation, distribution and reproduction in any medium or format, as long as you give appropriate credit to the original author(s) and the source, provide a link to the Creative Commons licence, and indicate if changes were made. The images or other third party material in this article are included in the article's Creative Commons licence, unless indicated otherwise in a credit line to the material. If material is not included in the article's Creative Commons licence and your intended use is not permitted by statutory regulation or exceeds the permitted use, you will need to obtain permission directly from the copyright holder. To view a copy of this licence, visit http://creativecommons.org/licenses/by/4.0/. The Creative Commons Public Domain Dedication waiver (http://creativeco mmons.org/publicdomain/zero/1.0/) applies to the data made available in this article, unless otherwise stated in a credit line to the data. 
Table 1 Comparison between meningococcal $(n=195)$ and pneumococcal $(n=67)$ purpura fulminans

\begin{tabular}{|c|c|c|c|}
\hline & $\begin{array}{l}\text { Meningococcal purpura } \\
\text { fulminans } n=195\end{array}$ & $\begin{array}{l}\text { Pneumococcal purpura } \\
\text { fulminans } n=67\end{array}$ & $p$ value \\
\hline \multicolumn{4}{|l|}{ Patient's characteristics and ICU scores } \\
\hline Male gender & $97(50)$ & $37(55)$ & 0.527 \\
\hline Age, years & 24 [19-45] & $49[38-60]$ & $<0.001$ \\
\hline SAPS $\|$ & $50[35-66]$ & $63[58-72]$ & $<0.001$ \\
\hline SOFA & $11[8-14]$ & $14[11-15]$ & $<0.001$ \\
\hline \multicolumn{4}{|l|}{ Main comorbidities } \\
\hline Chronic alcoholism & $5(2)$ & $9(13)$ & 0.002 \\
\hline Diabetes mellitus & $3(2)$ & $4(6)$ & 0.073 \\
\hline Asplenia or hyposplenia & $3(2)$ & $34(51)$ & $<0.001$ \\
\hline Malignant hemopathy & $1(1)$ & $2(3)$ & 0.162 \\
\hline Chronic respiratory disease & $18(23)$ & $14(28)$ & 0.625 \\
\hline Immunocompromised status & $5(3)$ & $4(6)$ & 0.241 \\
\hline No coexisting comorbid conditions & $164(84)$ & $22(33)$ & $<0.001$ \\
\hline \multicolumn{4}{|l|}{ Clinical features upon ICU admission } \\
\hline Days between disease onset and ICU admission, days & $4[4-5]$ & $5[4-6]$ & 0.003 \\
\hline Headache & $99(51)$ & $26(39)$ & 0.121 \\
\hline Myalgia & $48(25)$ & $12(18)$ & 0.338 \\
\hline Digestive signs & $124(64)$ & $41(61)$ & 0.839 \\
\hline Coma Glasgow score & 15 [13-15] & 15 [13-15] & 0.751 \\
\hline Temperature, ${ }^{\circ} \mathrm{C}$ & $38.5[37-40]$ & $38.5[37-39]$ & 0.802 \\
\hline Neck stiffness & $52(27)$ & $6(9)$ & 0.004 \\
\hline Purpuric rash before ICU admission & $168(86)$ & $38(57)$ & $<0.001$ \\
\hline$\beta$-Lactam antibiotic therapy before ICU admission & $157(81)$ & $46(69)$ & 0.067 \\
\hline$\beta$-Lactam antibiotic therapy at ICU admission & $195(100)$ & $67(100)$ & - \\
\hline \multicolumn{4}{|l|}{ Biological data upon ICU admission } \\
\hline Leukocytes count, $10^{3} \mathrm{~mm}^{-3}$ & $10,700[4000-20,800]$ & $10,655[2500-19,750]$ & 0.717 \\
\hline Platelets count, $10^{3} \mathrm{~mm}^{-3}$ & $61,000[28,500-100,000]$ & $33,000[19,000-49,500]$ & $<0.001$ \\
\hline C-reactive protein, $\mathrm{g} / \mathrm{L}$ & 148 [90-247] & $179[141-289]$ & 0.095 \\
\hline Procalcitonin, $\mathrm{ng} / \mathrm{mL}$ & $48[14-100]$ & $102[55-164]$ & 0.087 \\
\hline Troponin, mg/L & $1[0.10-12]$ & $0.25[0.13-11]$ & 0.697 \\
\hline Creatine kinase, IU/L & 300 [110-852] & $812[365-3460]$ & 0.016 \\
\hline Serum urea, $\mathrm{mmol} / \mathrm{L}$ & $9[7-11]$ & 13 [11-15] & $<0.001$ \\
\hline Serum creatinine, $\mu \mathrm{moL} / \mathrm{L}$ & 190 [136-250] & $240[184-310]$ & $<0.001$ \\
\hline Prothrombin time, $\%$ & $33[22-44]$ & $29[15-38]$ & 0.227 \\
\hline Factor $V_{1} \%$ & 23 [10-49] & $21[9-29]$ & 0.246 \\
\hline Arterial lactate, $\mathrm{mmol} / \mathrm{L}$ & $7.40[5-11]$ & $8[6-11]$ & 0.798 \\
\hline Fibrinogen, $g / L$ & $1.70[0.6-3]$ & $1.16[0.5-2]$ & 0.122 \\
\hline \multicolumn{4}{|l|}{ Microbiological data at ICU admission } \\
\hline Bacteremia & $99(51)$ & $56(84$ & $<0.001$ \\
\hline Lumbar puncture performed & $125(64)$ & $29(43)$ & 0.004 \\
\hline Positive cerebro-spinal fluid culture & $72 / 125(58)$ & $11 / 29(38)$ & 0.080 \\
\hline \multicolumn{4}{|l|}{ Outcome in the ICU } \\
\hline Lowest LVEF, \% & $33[20-45]$ & $30[25-50]$ & 0.870 \\
\hline Inotropic agent & $91(64)$ & $35(61)$ & 0.894 \\
\hline Platelets transfusion & $57(29)$ & $46(69)$ & $<0.001$ \\
\hline Plasma transfusion & $67(34)$ & $44(66)$ & $<0.001$ \\
\hline Steroids for septic shock or meningitis & $116(60)$ & $45(67)$ & 0.333 \\
\hline Activated protein $C$ & $33(17)$ & $9(13)$ & 0.632 \\
\hline Invasive mechanical ventilation & $152(78)$ & $65(97)$ & 0.001 \\
\hline
\end{tabular}


Table 1 (continued)

\begin{tabular}{lllr}
\hline & $\begin{array}{l}\text { Meningococcal purpura } \\
\text { fulminans } \boldsymbol{n = 1 9 5}\end{array}$ & $\begin{array}{l}\text { Pneumococcal purpura } \\
\text { fulminans } \boldsymbol{n = 6 7}\end{array}$ & $\begin{array}{c}\boldsymbol{p} \text { value } \\
\text { Duration of tracheal intubation, days }\end{array}$ \\
Duration of vasopressors, days & $4[2-9]$ & $10[3-28]$ & $<0.001$ \\
Renal replacement therapy & $3[2-5]$ & $5[3-8]$ & $<0.001$ \\
Veno-arterial ECMO & $69(36)$ & $45(67)$ & $<(9)$ \\
Limb amputation & $7(4)$ & $21(31)$ & $<.001$ \\
Limb amputation among ICU survivors & $19(10)$ & $19 / 32(59)$ & 0.104 \\
Death in ICU & $18 / 125(14)$ & $35(52)$ & $<0.001$ \\
Duration of ICU stay, days & $70(36)$ & $14[3-35]$ & $<.001$ \\
Duration of hospital stay, days & $5[2-11]$ & $23[3-78]$ & $<0.001$ \\
\hline
\end{tabular}

Continuous variables are reported as median [Interquartile range] and compared between groups using the Student $t$-test. Categorical variables are reported as numbers (percentages) and compared using $x^{2}$ test. A $p$ value $<0.05$ was considered significant

ICU intensive care unit; IMV Invasive Mechanical Ventilation, ECMO Extracorporeal membrane oxygenation, LVEF Left ventricular ejection fraction, SAPSII Simplified Acute Physiology Score, SOFA Sequential Organ Failure Assessment

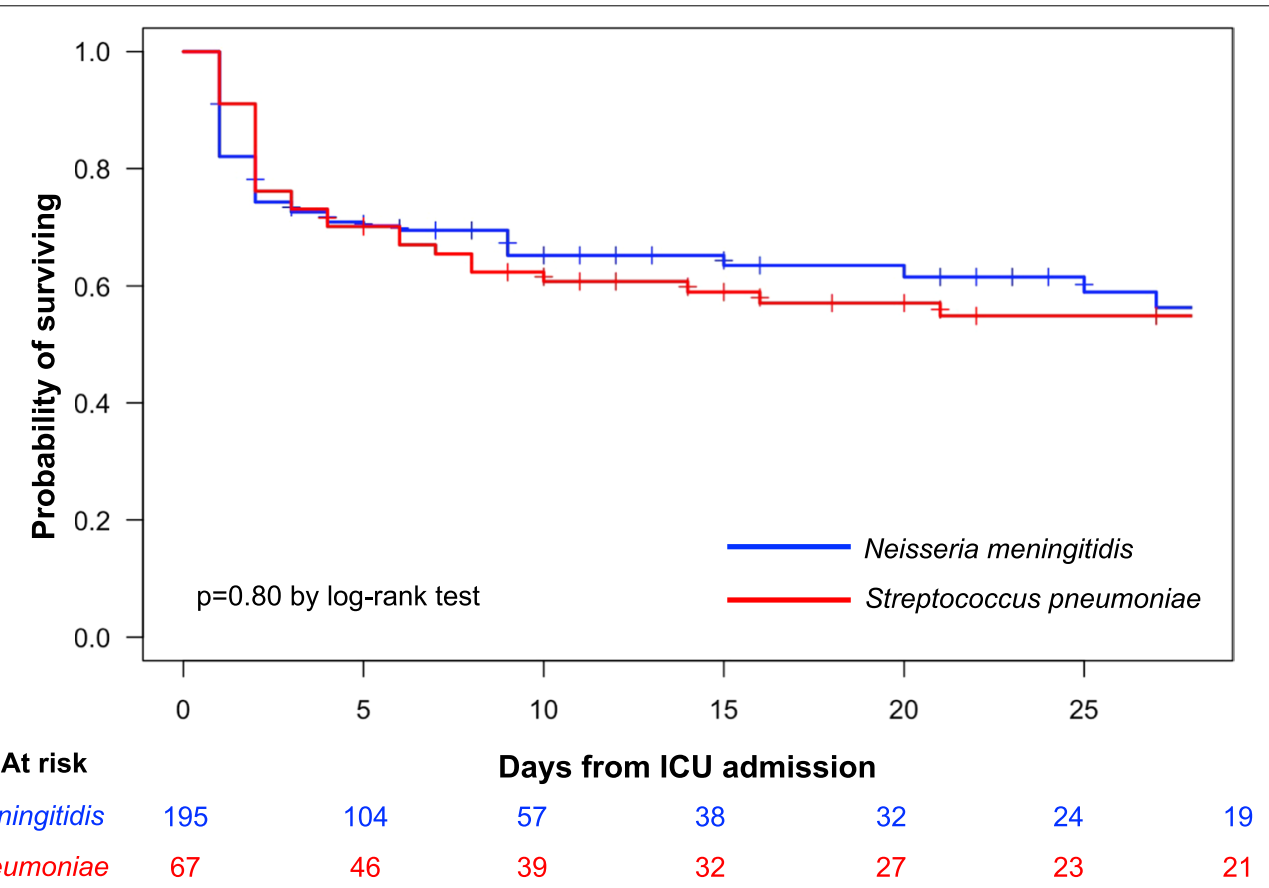

Fig. 1 Kaplan-Meier survival estimates during the 30 days following ICU admission of patients with pneumococcal (red curve) and meningococcal (blue curve) purpura fulminans

By multiple logistic regression adjusting on age, SOFA score, administration of $\beta$-lactam antibiotic therapy before ICU admission, platelet counts and arterial lactate levels, pPF was not associated with ICU mortality (adjusted Odds Ratio $=1.15$ 95\% CI 0.45-2.89, $p=0.77$ ).

As already reported in adults patients with bacterial meningitis [4], this study confirms that significant differences exist between $\mathrm{mPF}$ and $\mathrm{pPF}$, regarding both the clinical presentation at ICU admission and outcomes. Patients with pPF showed a different clinical phenotype, with less frequent purpura possibly leading to less frequent antibiotic treatment, more comorbidities with a more severe presentation at ICU admission, resulting in a higher rate of organ failures during ICU stay. Whether this more severe presentation should be ascribed to the level of virulence of the causative pathogen or to hostrelated characteristics is unsettled.

Our study has several limitations including its retrospective design and its long recruitment period with a high number of centers implying ICU procedures being 
inevitably heterogeneous. Nevertheless, the clinical presentation as well as the course in the ICU of patients with PF seem to differ according to the causative bacterium. This clinical observation should encourage researchers to better study the pathophysiology of pPF in order to develop targeted innovative therapies as being done for $\mathrm{mPF}[6]$.

\section{Abbreviations \\ ICU: Intensive care unit; mPF: Meningococcal purpura fulminans; pPF: Pneu- mococcal purpura fulminans; SOFA: Sequential Organ Failure Assessment.}

\section{Acknowledgements}

We thank the members of the HOPEFUL Study group (to be searchable through their individual PubMed records). Laurent Argaud (Lyon), François Barbier (Orléans), Amélie Bazire (Brest), Gaëtan Béduneau (Rouen), Frédéric Bellec (Montauban), Pascal Beuret (Roanne), Pascal Blanc (Pontoise), Cédric Bruel (Saint-Joseph), Christian Brun-Buisson (Mondor, AP-HP), Gwenhaël Colin (La Roche-sur-Yon), Delphine Colling (Roubaix), Alexandre Conia (Chartres), Rémi Coudroy (Poitiers), Martin Cour (Lyon), Damien Contou (Henri Mondor - AP-HP and Argenteuil), Fabrice Daviaud (Corbeil-Essonnes), Vincent Das (Montreuil), Jean Dellamonica (Nice), Nadège Demars (Antoine Beclère, AP-HP), Stephan Ehrmann (Tours), Arnaud Galbois (Quincy sous Sénart), Elodie Gelisse (Reims), Julien Grouille (Blois), Laurent Guérin (Ambroise Paré - AP-HP), Emmanuel Guérot (HEGP, AP-HP), Samir Jaber (Montpellier), Caroline Jannière (Créteil), Sébastien Jochmans (Melun), Mathieu Jozwiak (Kremlin Bicêtre, AP-HP), Pierre Kalfon (Chartres), Antoine Kimmoun (Nancy), Alexandre Lautrette (Clermont Ferrand), Jérémie Lemarié (Nancy), Charlène Le Moal (Le Mans), Christophe Lenclud (Mantes La Jolie), Nicolas Lerolle (Angers), Olivier Leroy (Tourcoing), Antoine Marchalot (Dieppe), Bruno Mégarbane (Lariboisière, AP-HP), Armand Mekontso Dessap (Mondor, AP-HP), Etienne de Montmollin (Saint-Denis), Frédéric Pène (Cochin, AP-HP), Claire Pichereau (Poissy), Gaëtan Plantefève (Argenteuil), Sébastien Préau (Lille), Gabriel Preda (Saint-Antoine, AP-HP), Nicolas de Prost (Henri Mondor, AP-HP), Jean-Pierre Quenot (Dijon), Sylvie Ricome (Aulnay-sous-Bois), Damien Roux (Louis Mourier, AP-HP), Bertrand Sauneuf (Cherbourg), Matthieu Schmidt (Pitié Salpétrière, AP-HP), Guillaume Schnell (Le Havre), Romain Sonneville (Bichat, AP-HP), Jean-Marc Tadié (Rennes), Yacine Tandjaoui (Avicenne, AP-HP), Martial Tchir (Villeneuve Saint Georges), Nicolas Terzi (Grenoble), Xavier Valette (Caen), Lara Zafrani (Saint-Louis, AP-HP), Benjamin Zuber (Versailles).

\section{Authors' contributions}

DC and NDP are responsible for the conception and design. All the authors were responsible for analysis and interpretation of data. All authors read, critically reviewed and approved the final manuscript. DC takes responsibility for the paper as a whole. All authors read and approved the final manuscript.

\section{Funding}

No funding

\section{Availability of data and materials}

The dataset used and analyzed for the current study is available from the corresponding author on reasonable request.

\section{Declarations}

\section{Ethics approval and consent to participate}

This study was conducted in accordance with the amended Declaration of Helsinki and was approved by the Institutional Review Board (CE 2016-01) of the French Intensive Care Society in March, 2016.

\section{Consent for publication}

Not applicable.

\section{Competing interests}

The authors declare that they have no competing interests.

\section{Author details}

1 Service de Réanimation Polyvalente, Centre Hospitalier Victor Dupouy, 69, rue du Lieutenant-Colonel Prudhon, 95100 Argenteuil, France. ${ }^{2}$ Service de Médecine Intensive Réanimation, Groupe de Recherche CARMAS, Centre Hospitalier Universitaire Henri Mondor, Assistance Publique-Hôpitaux de Paris, 51, avenue du Maréchal de Lattre de Tassigny, 94010 Créteil, France.

Received: 28 October 2021 Accepted: 4 November 2021

Published online: 11 November 2021

\section{References}

1. Contou D, Sonneville R, Canoui-Poitrine F, Colin G, Coudroy R, Pène F, et al. Clinical spectrum and short-term outcome of adult patients with purpura fulminans: a French multicenter retrospective cohort study. Intensive Care Med. 2018;44:1502-11.

2. Contou D, Mekontso Dessap A, de Prost N. Extracorporeal membrane oxygenation in adult patients with purpura fulminans. Crit Care Med. 2019;47:e1039-40.

3. Contou D, Canoui-Poitrine F, Coudroy R, Préau S, Cour M, Barbier F, et al. Long-term quality of life in adult patients surviving purpura fulminans: an exposed-unexposed multicenter cohort study. Clin Infect Dis. 2018;6:66.

4. van de Beek D, de Gans J, Spanjaard L, Weisfelt M, Reitsma JB, Vermeulen M. Clinical features and prognostic factors in adults with bacterial meningitis. N Engl J Med. 2004;351:1849-59.

5. Contou D, Coudroy R, Colin G, Tadié J-M, Cour M, Sonneville R, et al. Pneumococcal purpura fulminans in asplenic or hyposplenic patients: a French multicenter exposed-unexposed retrospective cohort study. Crit Care. 2020;24:68.

6. Denis K, Le Bris M, Le Guennec L, Barnier J-P, Faure C, Gouge A, et al. Targeting Type IV pili as an antivirulence strategy against invasive meningococcal disease. Nat Microbiol. 2019;4:972-84.

\section{Publisher's Note}

Springer Nature remains neutral with regard to jurisdictional claims in published maps and institutional affiliations.

Ready to submit your research? Choose BMC and benefit from:

- fast, convenient online submission

- thorough peer review by experienced researchers in your field

- rapid publication on acceptance

- support for research data, including large and complex data types

- gold Open Access which fosters wider collaboration and increased citations

- maximum visibility for your research: over 100M website views per year

At BMC, research is always in progress.

Learn more biomedcentral.com/submissions 